\title{
NUMERICAL SIMULATION OF NATURAL CONVECTION IN A POROUS CAVITY FILLED WITH FERROFLUID IN PRESENCE OF MAGNETIC SOURCE
}

\author{
B. Pekmen Geridönmez ${ }^{1^{*}}$
}

\begin{abstract}
In this study, numerical simulation of natural convection in a porous square cavity filled with $\mathrm{Fe}_{3} \mathrm{O}_{4}$-water is investigated. A magnetic source through the left wall of the cavity is also taken into account. Radial basis function based pseudo spectral (RBF-PS) method is applied. The effects of dimensionless parameters Darcy (Da), Hartmann (Ha), Rayleigh (Ra) numbers and solid volume fraction $\boldsymbol{\phi}$ are presented both in terms of streamlines, isotherms and vorticity contours and average Nusselt number through the heated wall. Convective heat transfer is inhibited with the rise of $\mathrm{Ha}$, and with the decrease in Da while it is enhanced with the increase in $\boldsymbol{\phi}$ and Ra.
\end{abstract}

\section{Keywords: Ferrofluid, Porous Media, Natural Convection, Radial Basis Functions, Magnetic Source}

\section{INTRODUCTION}

As a magnetic nanofluid, ferrofluids, are used in various fields such as electronic packing, mechanical engineering, thermal engineering, aerospace and bioengineering.

Nanofluids have been simulated in a lot of studies in different aspects. Khanafer; Vafai and Lightstone [1] analyzed heat transfer performance with a model considering the solid particle dispersion utilizing finite volume method (FVM) with alternating direction implicit procedure. They concluded that the increase in solid volume fraction at any Grashof number results in increase in heat transfer rate. Tiwari and Das [2] performed FVM to investigate mixed convection flow in a two sided lid-driven cavity filled with $\mathrm{Cu}$-water nanofluid. They reported that upward moving walls have a more reducing effect on heat transfer than the other ones. Muthtamilselvan; Kandaswamy and Lee [3] also employed FVM with SIMPLE algorithm on a staggered grid to investigate mixed convection flow in a two sided lid-driven cavity filled with $\mathrm{Cu}$-water nanofluid. Different aspect ratios are also observed, and they found that the average Nusselt number is linearly related with solid volume fraction. Sheremet; Oztop; Pop and Al-Salem [4] applied the second order finite difference method to simulate the free convection in an inclined wavy enclosure filled with a $\mathrm{Cu}$-water nanofluid under the effect of a uniform magnetic field and isothermal corner heater. Sheikholeslami [5] employed Runge-Kutta integration scheme considering Koo-Kleinstreuer-Li (KKL) model to analyze flow and heat transfer between two parallel plates one of which is heated, and the other is permeable. Results show that the Nusselt number has a direct relationship with power law index while has a reverse relation with expansion ratio. Turkyilmazoglu [6] presented a theoretical approach for obtaining the exact solutions of different nanofluids based with water concerning heat absorption, heat generation and radiation. The results show that heat transfer rate increases with heat absorption while heat generation and radiation have a reverse effect. A theory is also derived in closed form considering impacts of different types of nanofluids by Turkyilmazoglu [7]. Single phase and two phase models are investigated taking into account the hydrodynamic and thermal transport through the condensate film over curved walls. Using $\mathrm{LBM}, \mathrm{Al}_{2} \mathrm{O}_{3}$-water nanofluid modelled by KKL in a cubic cavity with moving bottom wall is simulated by Sheikholeslami; Hayat and Alsaedi [8]. Results reveal that nanofluid moves faster if nanoparticle concentration increases. Sheikholeslami and Rokni [9] applied Runge-Kutta method to two-phase nanofluid double diffusion convection considering induced magnetic field. Temperature gradient has a reverse relationship with Schmidt number, Brownian motion and thermopheretic parameters. Pekmen Geridonmez [10] solved natural convection flow in a cavity filled with nanofluid using RBFPS in space derivatives and differential quadrature method in time derivatives. In this study, both multi quadric (MQ) and inverse MQ RBF, and also different types of nanofluids are used.

Many researches have studied numerically and experimentally on ferrofluids in different geometries either in presence of external magnetic field or in absence of magnetic field. Tzirtzilakis and Xenos [11] investigated the blood flow in a lid-driven cavity under the effect of a localized magnetic field employing finite volume method 
(FVM) with SIMPLE algorithm. Their results showed that secondary vortices are formed in case of polarization. Aminfar; Mohammadpourfard and Ahangar Zonouzi [12] utilized the control volume technique with SIMPLEC for simulation of 3D laminar ferrofluid in presence of an electric current through a wire at the bottom of the duct resulting with transverse nonuniform magnetic field. Heat transfer enhancement is obtained in simulation of a water based ferrofluid in a mini channel using FVM in the study of Ghasemian; Ashrafi; Goharkhah and Ashjee [13]. Lattice Boltzmann method (LBM) is performed by Kefayati [14] to analyze the influence of heat dissipation and an external magnetic source on natural convection flow in a cavity filled with kerosene based cobalt. The increase in solid volume fraction of nanoparticles causes heat transfer to decrease at a large $\mathrm{Ra}=10^{5}$. Kefayati [15] used the same method to simulate the same problem in an inclined cavity. The effect of inclination angle on heat transfer is reduced with the increase in solid volume fraction. In studies [16]-[28], control volume based finite element method (CVFEM) is carried out for simulation. In the paper of Sheikholeslami and Ganji [16], governing equations based on ferrohydrodynamic (FHD) and magnetohydrodynamic (MHD) are solved in a semi annulus enclosure with sinusoidal hot wall with a magnetic source. At a low Rayleigh number, the effect of Kelvin force is pronounced. Natural convective heat transfer in a cavity with a circular step in which uniform heat flux is maintained is simulated under the effect of external variable magnetic field by Sheikholeslami [17]. In this study, MHD and FHD terms are added to governing equations. Sheikholeslami; Rashidi and Ganji [18] solve two phase model considering Brownian and thermopheresis in a lid-driven semi annulus under the effect of a magnetic source. The increase in Hartmann and Lewis number causes the Nusselt number to decrease while it increases with Reynolds number. The influence of magnetic field dependent viscosity is examined by Sheikholeslami and Rashidi [19] in a lid-driven semi annulus. Thermal radiation and viscosity of $\mathrm{Fe}_{3} \mathrm{O}_{4}$ as a function of magnetic field are taken into account by Sheikholeslami and Shehzad [20] in which an elliptic inner cylinder is settled into the enclosure. The results show that Nusselt number increases as inclination angle of the cylinder increases. Sheikholeslami; Ellahi and Vafai [21] showed that impact of adding $\mathrm{Fe}_{3} \mathrm{O}_{4}$ is more pronounced at a lower Rayleigh number. The radiation parameter is taken into account by Sheikholeslami [22] in which impact of a magnetic source in a square cavity including a circular hot cylinder is investigated. Results indicate that temperature gradient augments with the rise of radiation parameter $\mathrm{Rd}$ while temperature reduced with the augmentation of $\mathrm{Rd}$. Sheikholeslami also studied [23] free convection in a lid-driven cavity filled with $\mathrm{Fe}_{3} \mathrm{O}_{4}$-water. In this study, Columb forces resulting from the electric field on the cavity are also considered. Results report that average Nusselt number enhances with the rise of supplied voltage and the temperature gradient through the hot wall increases as Columb force augments. Ferrofluid flow in a curved cavity with constant heat flux condition for inner wall considering Rd parameter is presented by Sheikholeslami and Shamlooei [24]. Nanofluid velocity increases as Rd increases, and Rd is much more effective on convective heat transfer at large Rayleigh numbers. Sheikholeslami [25] added the radiation source term in energy equation for simulating the ferrofluid free convection concerning viscosity of ferrofluid in a cavity with centered elliptic cylinder. Inner wall temperature increases as Rd increases. Sheikholeslami and Shehzad [26] studied MHD convection in ferrofluid flow and heat transfer under the influence of a radiation source term. Viscosity of ferrofluid as a function of magnetic field is also considered in this study. In a half circular shaped cavity, Sheikholeslami; Hayat and Alsaedi [27] investigated ferrofluid with Kelvin forces in presence of external magnetic field. Results demonstrate that temperature gradient increases if Kelvin forces increase. Sheikholeslami and Rokni [28] showed the improvement of heat transfer with the increase in Lorentz forces considering a circular hot cylinder inside a cavity filled with ferrofluid.

Nanofluids in porous medium are encountered in [29]-[43]. Using FVM with SIMPLE algorithm, Malik and Nayak [29] solved Cu-water nanofluid with Darcy-Brinkmann-Forchheimer model considering the magnetic field. They reported that the rise in Darcy number augments the heat transfer rate. Sheikholeslami [30] conducted the fourth order Runge Kutta integration scheme featuring a shooting technique to analyze the nanofluid flow and heat transfer in a stretching porous cylinder. In this study, KKL model is adopted. Results shows that average Nusselt number increases as the suction parameter increases, and skin friction coefficient has a reverse relation with solid volume fraction. Impact of external magnetic source on free convection of ferrofluid in a porous curved cavity with hot left wall having constant heat flux condition is examined by Sheikholeslami [31] utilizing CVFEM. Sheikholeslami [32] also investigated free convection of ferrofluid with an external magnetic source in a porous curved cavity. In low Darcy number, adding nanoparticles enhances the heat transfer. Non-Darcy model in a nanofluid filled with $\mathrm{CuO}$ nanoparticles under the effect of a uniform magnetic field is studied by Sheikholeslami and Ganji [33] considering radiation effect. The results showed that the heat transfer rate increase with the increase in radiation paramater. Sheikholeslami and Bhatti [34] studied Brownian motion impact on viscosity of nanofluid 
and different shapes of $\mathrm{CuO}$ nanoparticles in a porous semi annulus with a moving bottom wall in presence of an uniform magnetic field. They also declared that average Nusselt number increases with the rise in Darcy number. Sheikholeslami [35] simulated the natural convection in a porous media filled with CuO-water and a sinusoidal wall under the effect of a constant magnetic field using Darcy's law and KKL model. Sheikholeslami [36] carried out CVFEM to investigate free convection in a ferrofluid-filled porous curved cavity in existence of magnetic source. In lower value of Darcy number, adding nanoparticles is more meaningful. Sheikholeslami [37] also applied LBM to examine the effect of magnetic field in an open porous cavity filled with CuO-water nanofluid. LBM is also utilized by Sheikholeslami [38] in order to simulate 3D forced convection in a porous, lid-driven cubic cavity. Outputs demonstrate that temperature gradient over hot surface decreases as Hartmann number increases while it augments with the rise of Reynolds and Darcy numbers. In presence of constant magnetic field, natural convection flow in a porous cavity (in Darcy's law) involving centered square cylinder and CuO-water nanofluid (with KKL model) is investigated by Sheikholeslami [39]. Average Nusselt number increases as the size of the inner square cylinder increases. $\mathrm{CuO}$-water nanofluid (in different shapes of nanoparticles) forced convection in a porous semi-annulus under the effect of uniform magnetic field is reported by Sheikholeslami and Bhatti [40]. Results show that platelet shape has the highest rate of heat transfer. The same nanofluid in a porous semi annulus with sinusoidal hot wall and with constant heat flux is examined by Sheikholeslami and Shehzad [41] using KKL and Darcy's models. Adding nanoparticles are more efficient in case of high Hartmann numbers. In an elliptic porous enclosure including a rectangular hot cylinder, $\mathrm{CuO}$-water nanofluid natural convection is presented by Sheikholeslami and Zeeshan [42]. Heat transfer enhancement increases with the increase in rotation angle of the cylinder. 3D nanofluid non-Darcy natural convection with Lorentz forces is reported utilizing LBM by Sheikholeslami [43]. In results, convection dominates if Darcy number, Rayleigh number and solid volume fraction increase, and is reduced with the rise of Hartmann number.

In a technical note prepared by Oztop; Selimefendigil; Abu-Nada and Al-Salem [44], computational methods used in case of curvilinear boundaries are reviewed considering the natural convection in nanofluids. In view of an experimental study by Roy; Asirvatham; Kunhappan; Cephas and Wongsises [45], the efficiency of low-particle concentrated silver-water nanofluid is investigated in a solar flat-plate collector.

In the present study, natural convection flow in a porous $\mathrm{Fe}_{3} \mathrm{O}_{4}$-water-filled cavity which has a magnetic source is investigated. The Brinkman-extended Darcy model is simulated utilizing the radial basis function based pseudo spectral (RBF-PS) method with multi quadric (MQ) RBFs. The effects of physical non-dimensional parameters are analyzed. To the author's knowledge, this method is firstly applied to this problem.

\section{PHYSICAL AND MATHEMATICAL SETUP}

Two-dimensional, unsteady, laminar, incompressible flow in a porous square cavity filled with ferrofluid is taken into account. The constant thermophysical properties of the fluid are considered except the density variation treated by Boussinessq approximation. The porous medium is homogeneous, isotropic and the fluid and solid of porous medium are in thermal equilibrium. The radiation, induced electric current and displacement currents are neglected.

The problem configuration is depicted in Figure 1. A magnetic source close to the left hot wall $\left(T_{h}=1\right)$ is placed. Right wall is the cold wall $\left(\mathrm{T}_{\mathrm{c}}=0\right)$. Top and bottom walls are adiabatic $(\partial T / \partial n=0)$. No-slip boundary conditions for velocity $(\mathrm{u}=\mathrm{v}=0)$ are imposed.

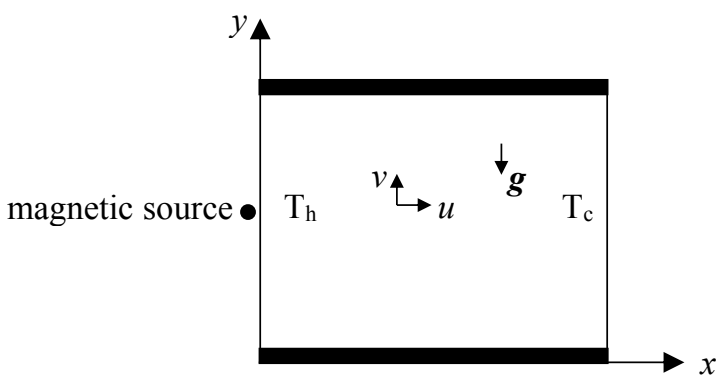

Figure 1. Problem configuration.

Thermophysical properties of water and magnetite $\mathrm{Fe}_{3} \mathrm{O}_{4}$ are given in Table 1. 
Journal of Thermal Engineering, Research Article, Vol. 4, No. 2, Special Issue 7, pp. 1756-1769,

February, 2018

Table 1. Physical Properties

\begin{tabular}{|c|c|c|c|c|c|}
\hline & $\boldsymbol{\rho}\left(\boldsymbol{k g} / \boldsymbol{m}^{\mathbf{3}}\right)$ & $\boldsymbol{c}_{\boldsymbol{p}}\left(\frac{\boldsymbol{J}}{\boldsymbol{k g} \boldsymbol{K}}\right)$ & $\boldsymbol{k}(\boldsymbol{W} / \mathbf{m} \boldsymbol{K})$ & $\begin{array}{l}\boldsymbol{\beta} \times \mathbf{1 0}^{-\mathbf{5}} \\
(\mathbf{1} / \boldsymbol{K})\end{array}$ & $\boldsymbol{\sigma}\left(\frac{\boldsymbol{S}}{\boldsymbol{m}}\right)$ \\
\hline Water & 997.1 & 4179 & 0.613 & 21 & 0.05 \\
\hline $\mathrm{Fe}_{3} \mathbf{O}_{4}$ & 5200 & 670 & 6 & 1.3 & 25000 \\
\hline
\end{tabular}

The magnetic field intensity and its components are defined as

$$
\begin{gathered}
\bar{H}=\sqrt{\overline{H_{x}}+\overline{H_{y}}} \\
\overline{H_{x}}=\frac{\gamma}{2 \pi} \frac{y-\bar{b}}{(\bar{b}-y)^{2}+(\bar{a}-x)^{2}} \\
\overline{H_{y}}=\frac{\gamma}{2 \pi} \frac{x-\bar{a}}{(\bar{b}-y)^{2}+(\bar{a}-x)^{2}}
\end{gathered}
$$

where $(\bar{a}, \bar{b})$ is the location of the magnetic source and $\gamma$ is the strength of the magnetic field at the source.

The physical governing equations are [36]

$$
\begin{gathered}
\frac{\partial u}{\partial x}+\frac{\partial v}{\partial y}=0 \\
\frac{\mu_{n f}}{\rho_{n f}} \nabla^{2} u=\frac{\partial u}{\partial t}+u \frac{\partial u}{\partial x}+v \frac{\partial u}{\partial y}+\frac{1}{\rho_{n f}} \frac{\partial p}{\partial x}+\frac{\mu_{0}^{2} \sigma_{n f}}{\rho_{n f}}\left({\overline{H_{y}}}^{2} u-\overline{H_{x} H_{y}} v\right)+\frac{\mu_{n f}}{\kappa \rho_{n f}} u \\
\frac{\mu_{n f}}{\rho_{n f}} \nabla^{2} v=\frac{\partial v}{\partial t}+u \frac{\partial v}{\partial x}+v \frac{\partial v}{\partial y}+\frac{1}{\rho_{n f}} \frac{\partial p}{\partial y}+\frac{\mu_{0}^{2} \sigma_{n f}}{\rho_{n f}}\left({\overline{H_{x}}}^{2} v-\overline{H_{x} H_{y}} u\right)+\frac{\mu_{n f}}{\kappa \rho_{n f}} v \\
-g \beta_{n f}\left(T-T_{c}\right) \\
\frac{k_{n f}}{\left(\rho c_{p}\right)_{n f}} \nabla^{2} T=\frac{\partial T}{\partial t}+u \frac{\partial T}{\partial x}+v \frac{\partial T}{\partial y}-\frac{\mu_{0}^{2} \sigma_{n f}}{\left(\rho c_{p}\right)_{n f}}\left(\overline{H_{x}} v-\overline{H_{y}} u\right)^{2} \\
-\frac{\mu_{n f}}{\left(\rho c_{p}\right)_{n f}}\left[2\left(\frac{\partial u}{\partial x}\right)^{2}+2\left(\frac{\partial v}{\partial y}\right)^{2}+2\left(\frac{\partial u}{\partial y}+\frac{\partial v}{\partial x}\right)^{2}\right]
\end{gathered}
$$

where the physical parameters are given by Khanafer; Vafai and Lightstone [1], Brinkman [46] and MaxwellGarnett [47] as

$$
\begin{gathered}
\rho_{n f}=\rho_{f}(1-\phi)+\rho_{s} \\
\left(\rho c_{p}\right)_{n f}=\left(\rho c_{p}\right)_{f}(1-\phi)+\left(\rho c_{p}\right)_{s} \\
\mu_{n f}=\frac{\mu_{f}}{(1-\phi)^{2.5}} \\
\beta_{n f}=\beta_{f}(1-\phi)+\beta_{s} \\
\alpha_{n f}=\frac{k_{n f}}{\left(\rho c_{p}\right)_{n f}}
\end{gathered}
$$


Journal of Thermal Engineering, Research Article, Vol. 4, No. 2, Special Issue 7, pp. 1756-1769,

February, 2018

$$
\begin{gathered}
k_{n f}=k_{f}\left(\frac{k_{s}-2 \phi\left(k_{f}-k_{s}\right)+2 k_{f}}{k_{s}+\phi\left(k_{f}-k_{s}\right)+2 k_{f}}\right) \\
\sigma_{n f}=\sigma_{f}\left(\frac{3\left(\frac{\sigma_{s}}{\sigma_{f}}-1\right) \phi}{\left(\frac{\sigma_{s}}{\sigma_{f}}+2\right)-\left(\frac{\sigma_{s}}{\sigma_{f}}-1\right) \phi}+1\right)
\end{gathered}
$$

and $\mu_{0}$ is the magnetic permeability of vacuum.

In order to obtain dimensionless governing equations, the non-dimensional parameters are defined as

$$
\begin{aligned}
\left(a^{\prime}, b^{\prime}\right)=\frac{(\bar{a}, \bar{b})}{L}, \quad\left(x^{\prime}, y^{\prime}\right)=\frac{(x, y)}{L}, \quad\left(H_{x}^{\prime}, H_{y}^{\prime}, H^{\prime}\right)=\frac{\left(\overline{H_{x}}, \overline{H_{y}}, \bar{H}\right)}{\overline{H_{0}}}, \\
p^{\prime}=\frac{p L^{2}}{\rho_{f} \alpha_{f}^{2}}, \quad u^{\prime}=\frac{u L}{\alpha_{f}}, \quad v^{\prime}=\frac{v L}{\alpha_{f}}, \quad T^{\prime}=\frac{T-T_{c}}{T_{h}-T_{c}}
\end{aligned}
$$

where $\overline{H_{0}}=\bar{H}(\bar{a}, 0)=\frac{\gamma}{2 \pi|b|}$, and $L$ is the characteristic length. These parameters are put into the dimensional equations Eq.(4)-(7). Then, the prime notations are dropped and dimensionless equations in $u-v-p-T$ form are derived as

$$
\begin{gathered}
\frac{\partial u}{\partial x}+\frac{\partial v}{\partial y}=0 \\
\nabla^{2} u=\frac{1}{\operatorname{Pr}} \frac{\rho_{n f} \mu_{f}}{\rho_{f} \mu_{n f}}\left(\frac{\partial u}{\partial t}+u \frac{\partial u}{\partial x}+v \frac{\partial u}{\partial y}\right)+\frac{\partial p}{\partial x}+H a^{2} \frac{\sigma_{n f} \mu_{f}}{\sigma_{f} \mu_{n f}}\left(H_{y}^{2} u-H_{x} H_{y} v\right)+\frac{u}{D a} \\
\nabla^{2} v=\frac{1}{\operatorname{Pr}} \frac{\rho_{n f} \mu_{f}}{\rho_{f} \mu_{n f}}\left(\frac{\partial v}{\partial t}+u \frac{\partial v}{\partial x}+v \frac{\partial v}{\partial y}\right)+\frac{\partial p}{\partial y}+H a^{2} \frac{\sigma_{n f} \mu_{f}}{\sigma_{f} \mu_{n f}}\left(H_{x}^{2} v-H_{x} H_{y} u\right)+\frac{v}{D a} \\
-R a \frac{\rho_{n f} \mu_{f} \beta_{n f}}{\rho_{f} \mu_{n f} \beta_{f}} \frac{\partial T}{\partial x} \\
\nabla^{2} T=\frac{\left(\rho c_{p}\right)_{n f} k_{f}}{\left(\rho c_{p}\right)_{f} k_{n f}}\left(\frac{\partial T}{\partial t}+u \frac{\partial T}{\partial x}+v \frac{\partial T}{\partial y}\right)-H a^{2} E c \frac{\sigma_{n f} k_{f}}{\sigma_{f} k_{n f}}\left(H_{x} v-H_{y} u\right)^{2} \\
-E c \frac{\mu_{n f} k_{f}}{\mu_{f} k_{n f}}\left[2\left(\frac{\partial u}{\partial x}\right)^{2}+2\left(\frac{\partial v}{\partial y}\right)^{2}+2\left(\frac{\partial u}{\partial y}+\frac{\partial v}{\partial x}\right)^{2}\right]
\end{gathered}
$$

in which the dimensionless parameters Prandtl, Hartmann, Darcy, Rayleigh and Eckert numbers, respectively, are

$$
\operatorname{Pr}=\frac{v_{f}}{\alpha_{f}}, \quad H a=L \mu_{0} \overline{H_{0}} \sqrt{\frac{\sigma_{f}}{\mu_{f}}}, \quad D a=\frac{\kappa}{L^{2}}, \quad R a=\frac{g \beta_{f} L^{3} \Delta T}{\alpha_{f} v_{f}}, \quad E c=\frac{\mu_{f} \alpha_{f}}{L^{2}\left(\rho c_{p}\right)_{f} \Delta T}
$$

The velocity components in terms of stream function $\psi(u=\partial \psi / \partial y, v=-\partial \psi / \partial x)$, provide automatically satisfaction of continuity equation, and pressure terms are eliminated applying the definition of vorticity $\omega=\nabla \times \boldsymbol{u}$ to the momentum equations. Then, in addition to the dimensionless energy equation Eq.(19) stream function and vorticity equations are deduced as

$$
\nabla^{2} \psi=-\omega
$$


Journal of Thermal Engineering, Research Article, Vol. 4, No. 2, Special Issue 7, pp. 1756-1769,

February, 2018

$$
\begin{aligned}
\nabla^{2} \omega=\frac{1}{\operatorname{Pr}} \frac{\rho_{n f} \mu_{f}}{\rho_{f} \mu_{n f}} & \left(\frac{\partial \omega}{\partial t}+u \frac{\partial \omega}{\partial x}+v \frac{\partial \omega}{\partial y}\right)+\frac{\omega}{D a}-R a \frac{\rho_{n f} \mu_{f} \beta_{n f}}{\rho_{f} \mu_{n f} \beta_{f}} \frac{\partial T}{\partial x} \\
+ & H a^{2} \frac{\sigma_{n f} \mu_{f}}{\sigma_{f} \mu_{n f}}\left[2 H_{x} \frac{\partial H_{x}}{\partial x} v-\frac{\partial H_{x}}{\partial x} H_{y} u-H_{x} \frac{\partial H_{y}}{\partial x} u+H_{x}^{2} \frac{\partial v}{\partial x}-H_{x} H_{y} \frac{\partial u}{\partial x}\right. \\
& \left.-2 H_{y} \frac{\partial H_{y}}{\partial y} u-H_{y}^{2} \frac{\partial u}{\partial y}+\frac{\partial H_{x}}{\partial y} H_{y} v+H_{x} \frac{\partial H_{y}}{\partial y} v+H_{x} H_{y} \frac{\partial v}{\partial y}\right]
\end{aligned}
$$

\section{NUMERICAL APPROACH}

Pseudo spectral (PS) methods are global and high accurate methods. Radial basis functions based pseudo spectral (RBF-PS) method is based on the approximation of all derivatives in the problem by RBFs. Radial basis functions enable one to use any type of grids, and are attracted by most of the researchers in mesh-free methods. The novel books by Fasshauer [48] and Fasshauer and McCourt [49] involve both theory and applications on RBFs.

RBFs approximate an unknown $\varphi(\psi, T$ or $\omega)$ in a diffusion-convection type equation, $\nabla^{2} \varphi=u \cdot \nabla \varphi$, as

$$
\varphi_{i}=\sum_{j=1}^{N_{b}+N_{i}} \alpha_{j} f_{i j}
$$

where $N_{b}$ is the number of boundary nodes, $N_{i}$ is the number of interior nodes, $f$ 's are RBFs depending on radial distance $r=\left\|\boldsymbol{x}-x_{j}\right\|\left(\boldsymbol{x}=(x, y)\right.$ is the field point and $\boldsymbol{x}_{j}=\left(x_{j}, y_{j}\right)$ is the collocation point), and $\alpha_{j}$ 's are initially unknown coefficients.

Eq.(23) can also be expressed in matrix-vector form as

$$
\varphi=F \alpha
$$

where the matrix $F$ is of size $\left(N_{b}+N_{i}\right) \times\left(N_{b}+N_{i}\right)$, and the coefficient vector $\alpha$ is $\left\{\alpha_{1}, \ldots, \alpha_{N_{b}+N_{i}}\right\}$. Equivalently, $\alpha=F^{-1} \varphi$.

Using Eq.(23) and Eq.(24), the first and second order derivatives of $\varphi$ are derived as

$$
\begin{gathered}
\frac{\partial \varphi}{\partial x}=\frac{\partial F}{\partial x} \alpha=\frac{\partial F}{\partial x} F^{-1} \varphi, \quad \frac{\partial \varphi}{\partial y}=\frac{\partial F}{\partial y} \alpha=\frac{\partial F}{\partial y} F^{-1} \varphi \\
\frac{\partial^{2} \varphi}{\partial x^{2}}=\frac{\partial}{\partial x}\left(\frac{\partial \varphi}{\partial x}\right)=\frac{\partial^{2} F}{\partial x^{2}} F^{-1} \varphi, \quad \frac{\partial^{2} \varphi}{\partial y^{2}}=\frac{\partial^{2} F}{\partial y^{2}} F^{-1} \varphi
\end{gathered}
$$

RBF based pseudo spectral (PS) method for the space derivatives is carried out to simulate the equations (19), (21), (22). Backward Euler in time derivative is used.

An iterative system with the application of the method to the system is built as follows

$$
\begin{gathered}
D_{2} \psi^{n+1}=-\omega^{n} \\
\left(D_{2}-C_{1} \frac{\mathrm{I}}{\Delta t}-C_{1} M\right)=-C_{1} \frac{T^{n}}{\Delta t}-C_{2} H a^{2} E c\left(H_{x} v-H_{y} u\right)^{2} \\
-C_{3} E c\left[2\left(D_{x} u\right)^{2}+2\left(D_{y} v\right)^{2}+\left(D_{y} u+D_{x} v\right)^{2}\right] \\
\left(D_{2}-\frac{C_{4}}{P r} \frac{\mathrm{I}}{\Delta t}-\frac{C_{4}}{P r} M-\frac{\mathrm{I}}{D a}\right) \omega^{n+1}=-\frac{C_{4}}{P r} \frac{w^{n}}{\Delta t}-C_{5} R a D_{x} T^{n+1}+ \\
C_{6} H a^{2}\left[2 H_{x}\left(D_{x} H_{x}\right) v-\left(D_{x} H_{x}\right) H_{y} u-H_{x}\left(D_{x} H_{y}\right) u+H_{x}^{2}\left(D_{x} v\right)-H_{x} H_{y}\left(D_{x} u\right)\right. \\
\left.-2 H_{y}\left(D_{y} H_{y}\right) u-H_{y}^{2}\left(D_{y} u\right)+\left(D_{y} H_{x}\right) H_{y} v+H_{x}\left(D_{y} H_{y}\right) v+H_{x} H_{y}\left(D_{y} v\right)\right]
\end{gathered}
$$

where $n$ is the number of iteration, $C_{1}, C_{2}, C_{3}, C_{4}, C_{5}, C_{6}$ are constants seen in Eqs.(19), (21), (22), I is the identity matrix of size $\left(N_{b}+N_{i}\right) \times\left(N_{b}+N_{i}\right)$ and matrices 


$$
D_{2}=\left(\frac{\partial^{2} F}{\partial x^{2}}+\frac{\partial^{2} F}{\partial y^{2}}\right) F^{-1}, \quad D_{x}=\frac{\partial F}{\partial x} F^{-1}, \quad D_{y}=-\frac{\partial F}{\partial y} F^{-1}, \quad M=[u]_{d} D_{x}+[v]_{d} D_{y}
$$

Initially, stream function, temperature and vorticity are taken as zero everywhere in the computational domain except on the boundary. After solving Eq.(27), the velocity components $u=u^{n+1}$ and $v=v^{n+1}$ are computed by $u=D_{y} \psi^{n+1}, v=-D_{x} \psi^{n+1}$. Then, the boundary conditions for $u$ and $v$ are inserted. $u^{n+1}$ and $v^{n+1}$ are used in calculation of $M$ matrix in Eqs.(28) and (29). Once Eq.(29) is solved, the unknown vorticity boundary conditions are derived by using the definition of vorticity as $\omega=D_{x} v^{n+1}-D_{y} u^{n+1}$, and a relaxation parameter $\tau(0<\tau<1)$ is used as $\omega^{n+1} \leftarrow \tau \omega^{n+1}+(1-\tau) \omega^{n}$.

The iterations continue until the criterion

$$
\sum_{k=1}^{3} \frac{\left\|\varphi_{k}^{n+1}-\varphi_{k}^{n}\right\|_{\infty}}{\left\|\varphi_{k}^{n+1}\right\|_{\infty}}<10^{-5}
$$

is satisfied in which $\varphi_{1}=\psi, \varphi_{2}=T, \varphi_{3}=\omega$.

The average Nusselt number through the left hot wall is computed by

$$
\overline{N u}=-\frac{k_{n f}}{k_{f}} \int_{0}^{1} \frac{\partial T}{\partial x} d y
$$

\section{NUMERICAL RESULTS}

The multiquadric radial basis function $f=\sqrt{r^{2}+c^{2}}$ is used. As a difference from the study of Geridonmez Pekmen [10], in computation of $\left(\frac{\partial^{2} F}{\partial x^{2}}+\frac{\partial^{2} F}{\partial y^{2}}\right)$ in diffusion terms, a perturbed $r^{2}$ as $\sqrt{r^{2}+\varepsilon^{2}}$, in which $0<\varepsilon=c<1$, is tried. Because of this trial, the determination of shape parameter $c$ is also done differently.

In this study, the shape parameter $c$ is determined by taking the average value of minimum of absolute value of $x$ coordinate distances (different than zero) from interior nodes to all nodes. This determination is done with regard to the node distribution given in Figure 2. In this distribution, boundary nodes are Gauss-ChebyshevLobatto nodes (from $x_{1}$ to $x_{N}$ in which $N_{b}=4 N-4$ for unit square), and interior nodes are the Chebyshev nodes between $0.5\left(x_{2}+x_{3}\right)$ and $0.5\left(x_{N-2}+x_{N-1}\right)$.

The base fluid is taken as pure water with Prandtl number 6.8, and Eckert number and the time increment are fixed at $E c=10^{-5}, \Delta t=0.1$, respectively. The other dimensionless parameters are in the following ranges: $10^{-4} \leq D a \leq 10,10 \leq H a \leq 50,10^{3} \leq R a \leq 10^{5}, 0.04 \leq \phi \leq 0.2$. Since the inertial effects are not taken into account in this study, these ranges are consistent with the physical aspect as used in the study of Ramakrishna; Basak; Roy and Pop [50].

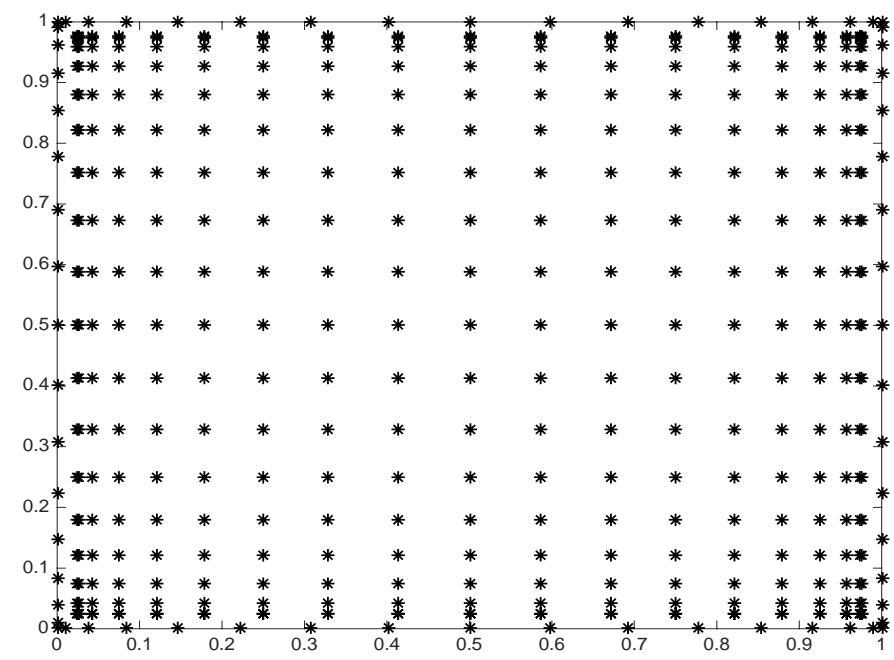

Figure 2. Node distribution. 
The validation of the method is shown in Table 2 comparing the average Nusselt number through the heated left wall. The results are in good agreement with the benchmark problem. One of the reasoning in the discrepancies in the results is to use small number of grid points, and the other one may be using the perturbation idea only in diffusion terms.

Table 2. Comparison with a benchmark problem.

\begin{tabular}{|c|c|c|c|c|c|}
\hline Ra & Davis [51] & Present & $\boldsymbol{N}_{\boldsymbol{b}}, \boldsymbol{N}_{\boldsymbol{i}}$ & $\boldsymbol{c}$ & $\Delta \boldsymbol{t}, \boldsymbol{\tau}$ \\
\hline $10^{3}$ & 1.117 & 1.1126 & 64,361 & 0.01964 & $0.1,0.1$ \\
\hline $10^{4}$ & 2.238 & 2.2673 & 80,529 & 0.01365 & $0.1,0.1$ \\
\hline $10^{5}$ & 4.509 & 4.5845 & 96,729 & 0.01017 & $0.1,0.1$ \\
\hline
\end{tabular}

A grid independence is searched for $R a=10^{4}$ and $R a=10^{5}$ in Table 3. $N_{b}=80, N_{i}=529$ for $R a=10^{4}$ and $N_{b}=120, N_{i}=1089$ for $R a=10^{5}$ are enough to visualize the problem. As noted, the values of $c$ are diminished by the rise in the number of nodes.

Table 3. Grid Independence investigation in $R a=10^{4}$ and $R a=10^{5}$.

\begin{tabular}{|c|c|c|c|}
\hline $\mathbf{R a}$ & $\overline{\mathbf{N u}}$ & $\boldsymbol{N}_{\boldsymbol{b}}, \boldsymbol{N}_{\boldsymbol{i}}$ & $\boldsymbol{c}$ \\
\hline $10^{4}$ & 2.0586 & 64,361 & 0.01964 \\
\hline & 2.0232 & 72,441 & 0.01615 \\
\hline & 1.9979 & 80,529 & 0.01365 \\
\hline & 1.9766 & 88,625 & 0.01170 \\
\hline & 1.9592 & 96,729 & 0.01070 \\
\hline & 1.9461 & 104,841 & 0.00897 \\
\hline $10^{5}$ & 3.4877 & 96,729 & 0.01070 \\
\hline & 3.4450 & 104,841 & 0.00897 \\
\hline & 3.4085 & 112,961 & 0.00801 \\
\hline & 3.3759 & 120,1089 & 0.0072 \\
\hline & 3.3453 & 128,1225 & 0.00652 \\
\hline
\end{tabular}

Figure 3 depicts the heat transfer and flow behavior in different $\mathrm{Ha}$ values. Primary vortex in streamlines is diminished pointing to the decrease in the fluid velocity. Also, the intense magnetic field pushes the flow from the left to the right. Isotherms almost become perpendicular to the top and bottom wall in which Lorentz force inhibits the heat transfer. In vorticity contours, the effect from the left wall via magnetic source is noticed as well as the combination of two vorticity vortexes in the center at a large $\mathrm{Ha}$.

The influence of Darcy number on heat transfer and fluid flow is described in Figure 4. As is seen, the decrease in porous permeability, a resistance to fluid flow is formed, and the effect of magnetic source on streamlines almost disappears. Isotherms show that the buoyancy-driven convection is pronounced at a large $D a$. Vorticity becomes stagnant at the center at a small $\mathrm{Da}$ due to the slow movement of fluid.

As expected in Figure 5, the increase in Rayleigh number emphasizes the natural convection, or convective heat transfer in other words. Strong temperature gradient and two elongated vortices in vorticity contours through the left and right walls are noted. The effect of magnetic source on streamlines is realized at a small $R a$ while it is reduced at $R a=10^{5}$.

The location of magnetic source is investigated in Figure 6. If the magnetic source is moved closer to the left wall when $b=0.5$ is fixed, streamlines and vorticity contours form a small peak on the left wall pointing to a small decrease in fluid flow. $a=1.05$ is a reverse case of $a=-0.05$ on the right wall. If the magnetic source is moved from bottom to top when $a=-0.05$ is fixed, the decrease in the convective heat transfer is exhibited on isotherms while there is no source effect in $b=0.05$. The primary vortex in streamlines is also decreased and moves a little bit to the right, and vorticity contours are unified and approached to the right when $b=0.75$. 
$\psi$
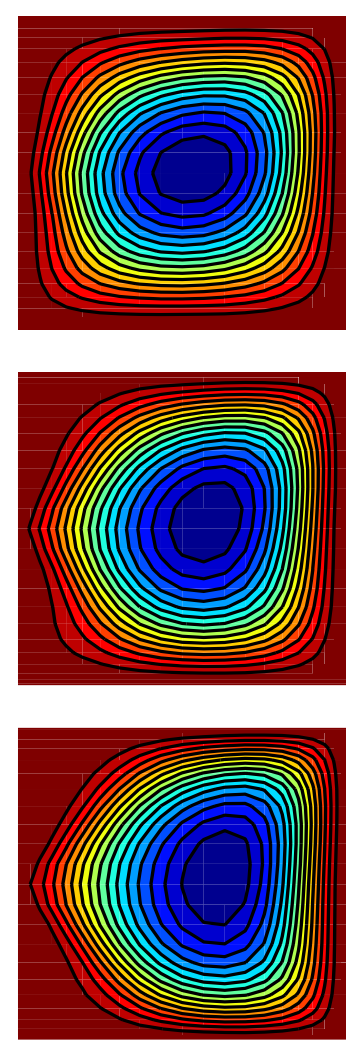

$\mathrm{T}$
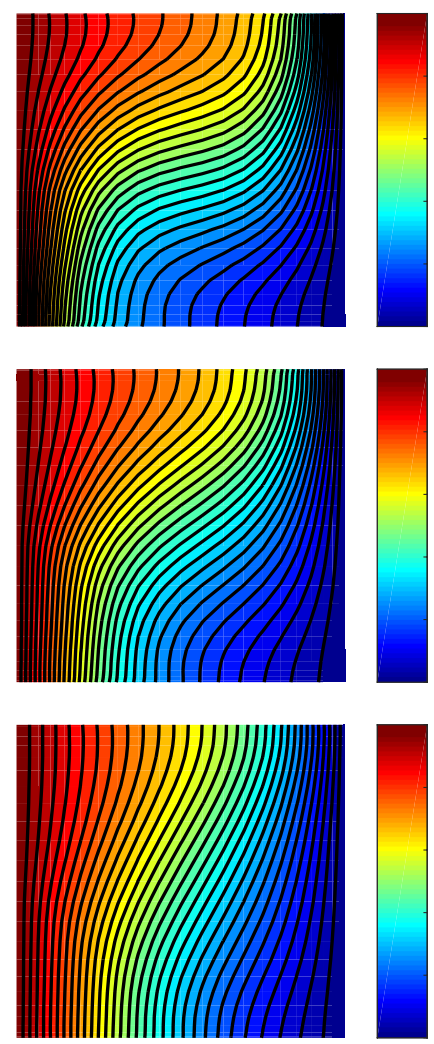

$\omega$
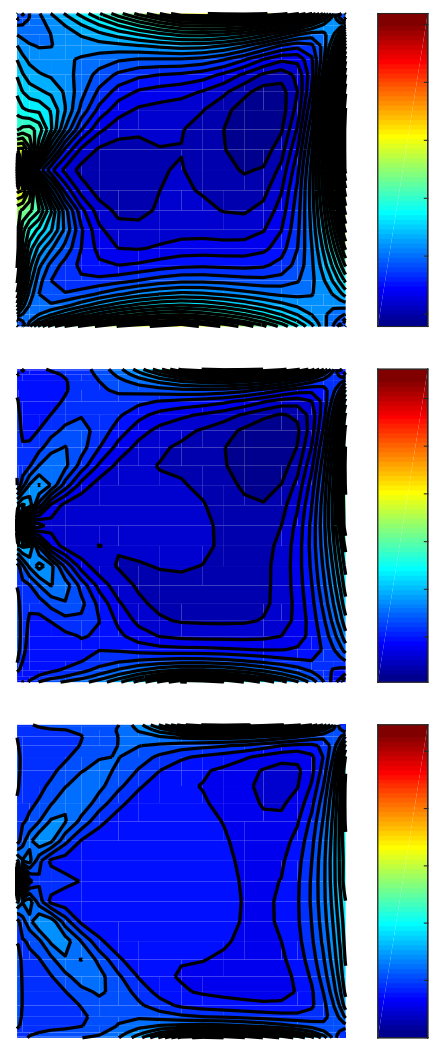

Figure 3. $H a$ variation when $D a=0.1, \phi=0.04, R a=10^{4}$.

Average Nusselt number values for the cases in Figures 3-6 are presented in Table 4. In each cases, convective heat transfer increases with the increase in concentration of nanoparticles. The augmentation in conductive heat transfer is investigated in small $\mathrm{Da}$ and large $\mathrm{Ha}$. Also, heat transfer is decelerated if the magnetic source is moved from left to right or from bottom to top.

Table 4. Average Nusselt number values in different $\phi$.

\begin{tabular}{|c|c|c|c|c|c|}
\hline & $\boldsymbol{\phi}=\mathbf{0 . 0 4}$ & $\boldsymbol{\phi}=\mathbf{0 . 0 8}$ & $\boldsymbol{\phi}=\mathbf{0 . 1 2}$ & $\boldsymbol{\phi}=\mathbf{0 . 1 6}$ & $\boldsymbol{\phi}=\mathbf{0 . 2}$ \\
\hline$D a=10^{-4}$ & 1.08 & 1.18 & 1.28 & 1.39 & 1.51 \\
\hline$D a=0.01$ & 1.64 & 1.71 & 1.77 & 1.83 & 1.89 \\
\hline$D a=10$ & 2.06 & 2.15 & 2.23 & 2.29 & 2.34 \\
\hline$H a=25$ & 1.53 & 1.61 & 1.68 & 1.75 & 1.82 \\
\hline$H a=50$ & 1.22 & 1.30 & 1.39 & 1.48 & 1.58 \\
\hline$a=-0.05$ & 2.00 & 2.09 & 2.16 & 2.22 & 2.27 \\
\hline$a=-0.01$ & 1.92 & 2.01 & 2.08 & 2.15 & 2.20 \\
\hline$a=1.05$ & 2.00 & 2.09 & 2.17 & 2.23 & 2.28 \\
\hline$b=0.05$ & 2.28 & 2.39 & 2.47 & 2.53 & 2.59 \\
\hline$b=0.25$ & 2.17 & 2.27 & 2.35 & 2.41 & 2.45 \\
\hline$b=0.75$ & 1.71 & 1.78 & 1.84 & 1.91 & 1.96 \\
\hline
\end{tabular}


Journal of Thermal Engineering, Research Article, Vol. 4, No. 2, Special Issue 7, pp. 1756-1769,

February, 2018

$\psi$
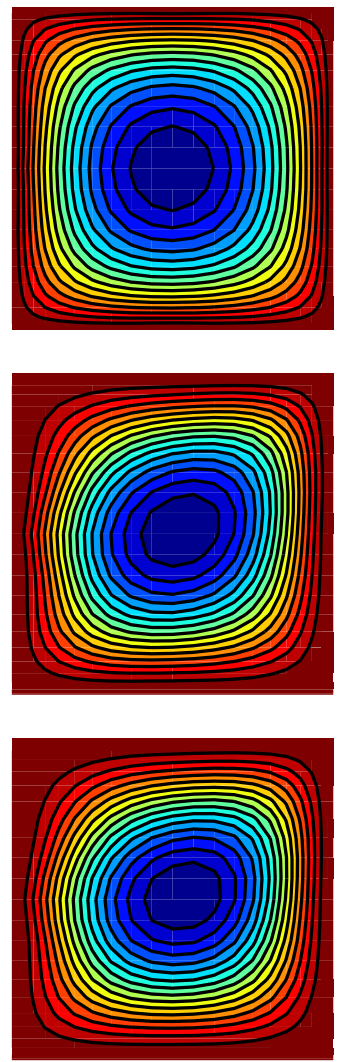

$\mathrm{T}$
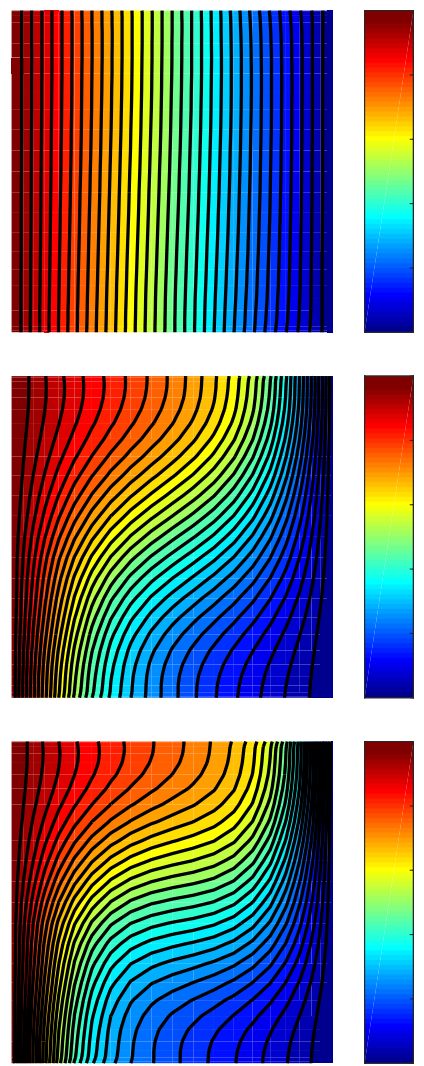
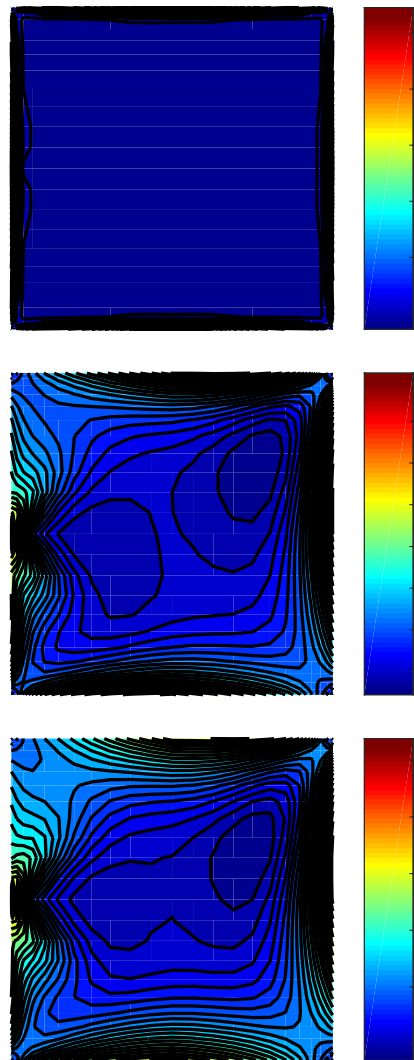

Figure 4. $D a$ variation when $H a=10, \phi=0.04, R a=10^{4}$.

$\psi$
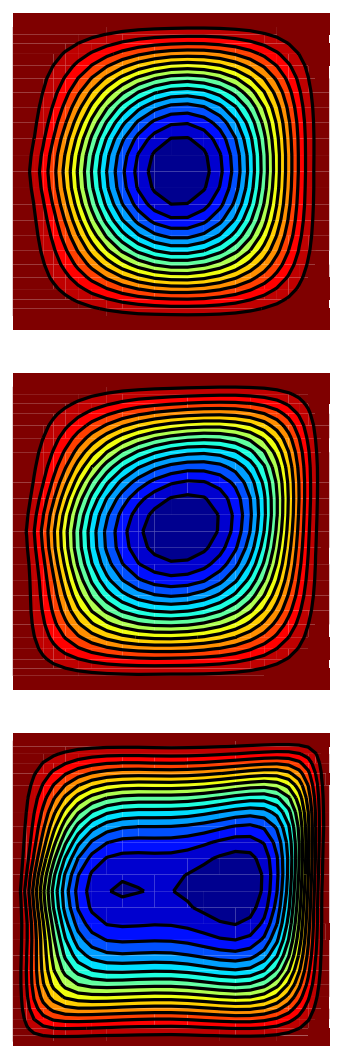

$\mathrm{T}$
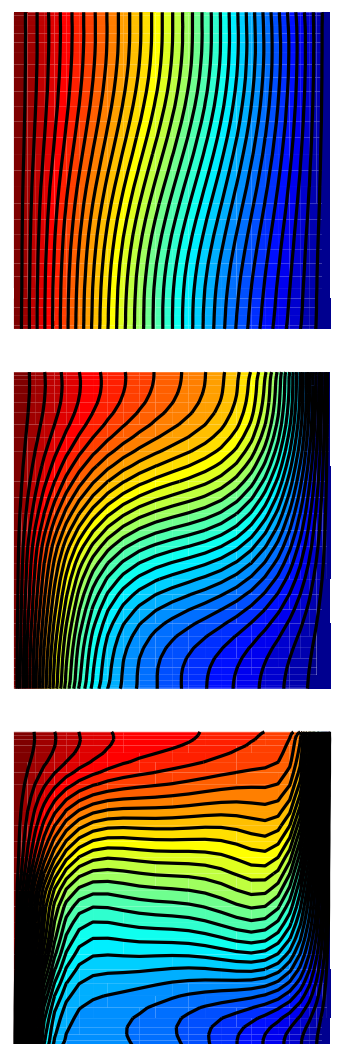

$\omega$
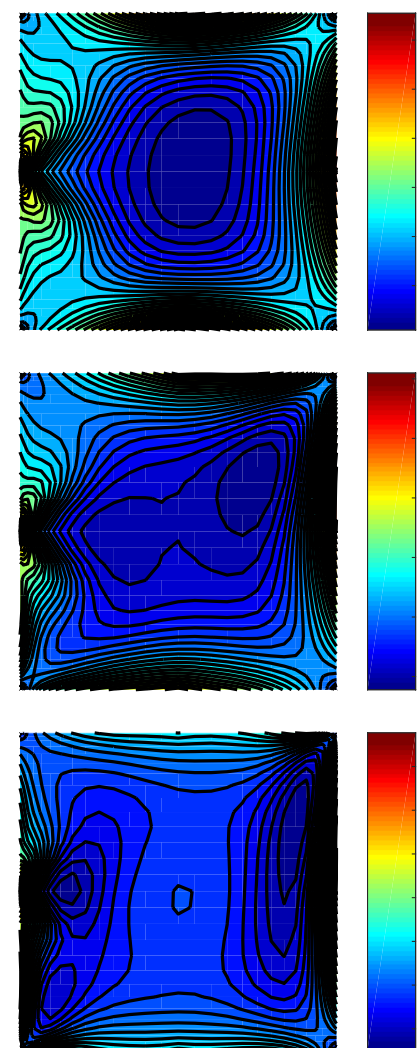

Figure 5. $R a$ variation when $D a=0.1, \phi=0.04, H a=10$. 

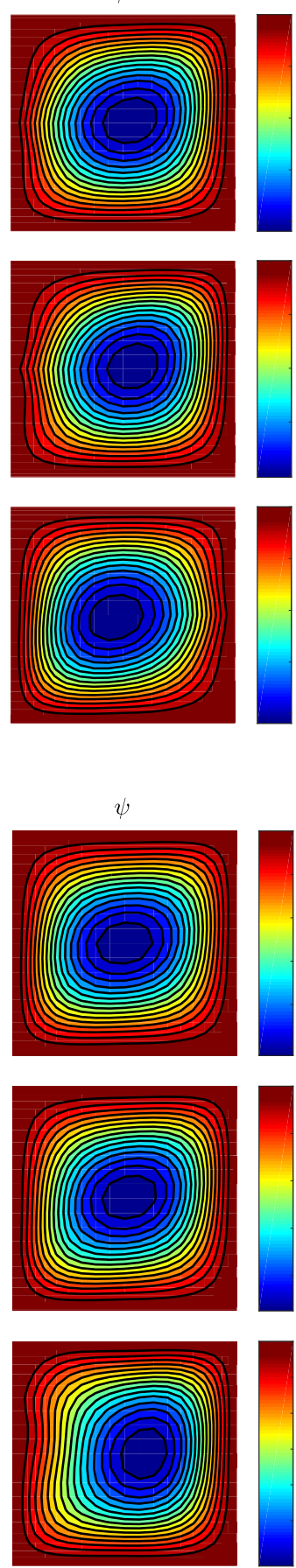

$\mathrm{T}$
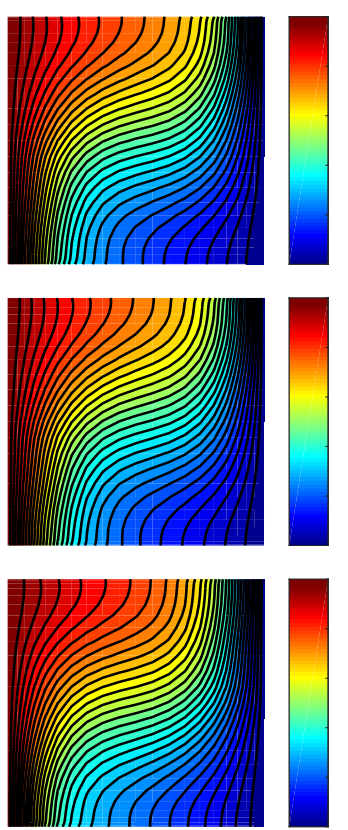

$\mathrm{T}$
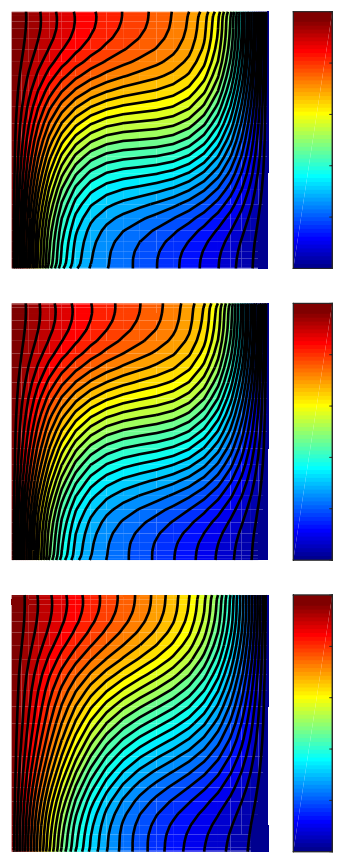

$\omega$
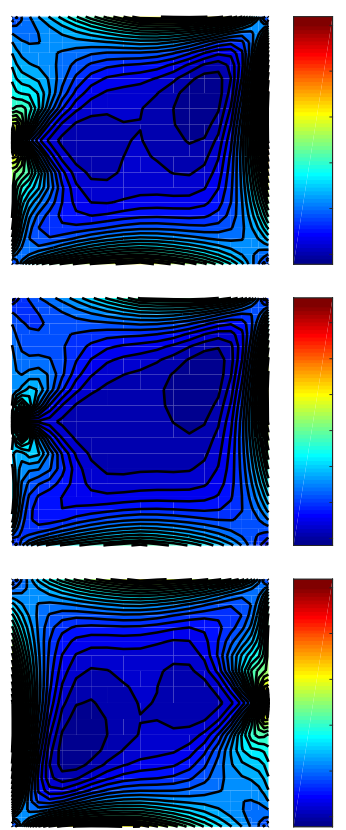

$\omega$
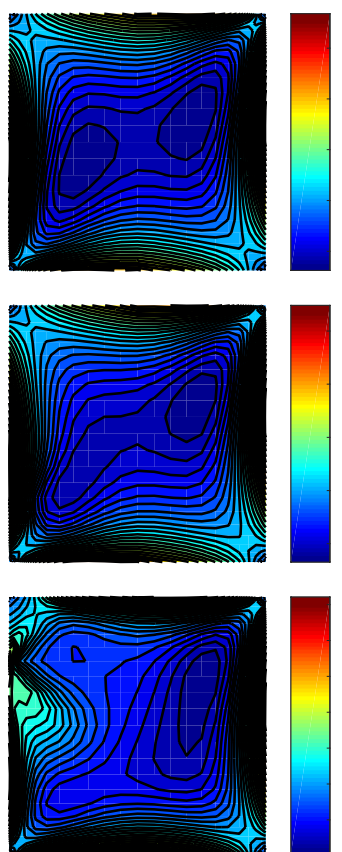

Figure 6. Location of magnetic source with $\mathrm{Da}=0.1, \phi=0.04, \mathrm{Ra}=10^{4}, \mathrm{Ha}=10$.

\section{CONCLUSION}

In this paper, RBF-PS method is carried out for simulation of natural convection in a porous cavity filled with ferrofluid and in presence of a magnetic source. The main goal of the current study is to examine the effect of Darcy number and the magnetic source. The convective heat transfer is declined with the decrease in Darcy number, and with the location of magnetic source close to the left wall or the top wall. The higher values of Hartmann number suppresses the fluid flow and heat transfer due to the retarding effect of Lorentz force while the large Rayleigh number and solid volume fraction value augment the convective heat transfer. Average Nusselt number augments with the rise of the concentration of the nanoparticles. RBF-PS method enable one to use small number of grid points. Also, the idea of the perturbed $r^{2}$ can also be extended to all matrices formed by $F$ in the iterative systems. 


\section{NOMENCLATURE}

$c_{p} \quad$ specific heat at constant pressure

Da Darcy number

Ec Eckert number

$H \quad$ strength of magnetic field

$H_{x} \quad$ x-component of $H$

$H_{y} \quad$ y-component of $H$

$\mathrm{Ha}$ Hartmann number

$k \quad$ thermal conductivity

$\mathrm{Pr} \quad$ Prandtl number

$\mathrm{Ra} \quad$ Rayleigh number

$T \quad$ fluid temperature

\section{Greek Symbols}

$\alpha \quad$ thermal diffusivity

$\beta \quad$ thermal expansion coefficient

$\kappa \quad$ permeability of the porous medium

$\mu \quad$ dynamic viscosity

$\mu_{0} \quad$ magnetic permeability of vacuum

$\phi \quad$ solid volume fraction

$\rho \quad$ density

$\sigma \quad$ electrical conductivity

\section{Subscripts}

$\begin{array}{ll}f & \text { base fluid } \\ n f & \text { nanofluid } \\ s & \text { solid } \\ c & \text { cold } \\ h & \text { hot }\end{array}$

\section{REFERENCES}

[1] Khanafer, K., Vafai, K., \& Lightstone, M. (2003). Buoyancy-driven heat transfer enhancement in a twodimensional enclosure utilizing nanofluids. International journal of heat and mass transfer, 46(19), 3639-3653.

[2] Tiwari, R. K., \& Das, M. K. (2007). Heat transfer augmentation in a two-sided lid-driven differentially heated square cavity utilizing nanofluids. International Journal of Heat and Mass Transfer, 50(9), 2002-2018.

[3] Muthtamilselvan, M., Kandaswamy, P., \& Lee, J. (2010). Heat transfer enhancement of copper-water nanofluids in a lid-driven enclosure. Communications in Nonlinear Science and Numerical Simulation, 15(6), 1501-1510.

[4] Sheremet, M. A., Oztop, H. F., Pop, I., \& Al-Salem, K. (2016). MHD free convection in a wavy open porous tall cavity filled with nanofluids under an effect of corner heater. International Journal of Heat and Mass Transfer, 103, 955-964.

[5] Kandelousi, M. S. (2014). KKL correlation for simulation of nanofluid flow and heat transfer in a permeable channel. Physics Letters A, 378(45), 3331-3339.

[6] Turkyilmazoglu, M. (2016). Natural convective flow of nanofluids past a radiative and impulsive vertical plate. Journal of Aerospace Engineering, 29(6), 04016049.

[7] Turkyilmazoglu, M. (2017). Condensation of laminar film over curved vertical walls using single and twophase nanofluid models. European Journal of Mechanics-B/Fluids, 65, 184-191.

[8] Sheikholeslami, M., Hayat, T., \& Alsaedi, A. (2017). Numerical simulation of nanofluid forced convection heat transfer improvement in existence of magnetic field using lattice Boltzmann method. International Journal of Heat and Mass Transfer, 108, 1870-1883..

[9] Sheikholeslami, M., \& Rokni, H. B. (2017). Nanofluid two phase model analysis in existence of induced magnetic field. International Journal of Heat and Mass Transfer, 107, 288-299. 
[10] Geridonmez, B. P. (2016). RBF simulation of natural convection in a nanofluid-filled cavity. AIMS Mathematics, 1(3), 195-207.

[11] Tzirtzilakis, E. E., \& Xenos, M. A. (2013). Biomagnetic fluid flow in a driven cavity. Meccanica, 48(1), 187200.

[12] Aminfar, H., Mohammadpourfard, M., \& Zonouzi, S. A. (2013). Numerical study of the ferrofluid flow and heat transfer through a rectangular duct in the presence of a non-uniform transverse magnetic field. Journal of Magnetism and Magnetic materials, 327, 31-42.

[13] Ghasemian, M., Ashrafi, Z. N., Goharkhah, M., \& Ashjaee, M. (2015). Heat transfer characteristics of Fe 3 $\mathrm{O} 4$ ferrofluid flowing in a mini channel under constant and alternating magnetic fields. Journal of Magnetism and Magnetic Materials, 381, 158-167.

[14] Kefayati, G. H. R. (2014). Natural convection of ferrofluid in a linearly heated cavity utilizing LBM. Journal of Molecular Liquids, 191, 1-9.

[15] Kefayati, G. R. (2014). Simulation of ferrofluid heat dissipation effect on natural convection at an inclined cavity filled with kerosene/cobalt utilizing the Lattice Boltzmann method. Numerical Heat Transfer, Part A: Applications, 65(6), 509-530.

[16] Sheikholeslami, M., \& Ganji, D. D. (2014). Ferrohydrodynamic and magnetohydrodynamic effects on ferrofluid flow and convective heat transfer. Energy, 75, 400-410.

[17] Kandelousi, M. S. (2014). Effect of spatially variable magnetic field on ferrofluid flow and heat transfer considering constant heat flux boundary condition. The European Physical Journal Plus, 129(11), 248.

[18] Sheikholeslami, M., Rashidi, M. M., \& Ganji, D. D. (2015). Numerical investigation of magnetic nanofluid forced convective heat transfer in existence of variable magnetic field using two phase model. Journal of Molecular Liquids, 212, 117-126.

[19] Sheikholeslami, M., \& Rashidi, M. M. (2015). Ferrofluid heat transfer treatment in the presence of variable magnetic field. The European Physical Journal Plus, 130(6), 115.

[20] Sheikholeslami, M., \& Shehzad, S. A. (2017). Thermal radiation of ferrofluid in existence of Lorentz forces considering variable viscosity. International Journal of Heat and Mass Transfer, 109, 82-92.

[21] Sheikholeslami, M., Ellahi, R., \& Vafai, K. (2017). Study of Fe 3 O 4-water nanofluid with convective heat transfer in the presence of magnetic source. Alexandria Engineering Journal.

[22] Sheikholeslami, M. (2016). Magnetic source impact on nanofluid heat transfer using CVFEM. Neural Computing and Applications, 1-10.

[23] Sheikholeslami, M. (2017). Influence of Coulomb forces on Fe $3 \mathrm{O} 4-\mathrm{H} 2 \mathrm{O}$ nanofluid thermal improvement. International Journal of Hydrogen Energy, 42(2), 821-829.

[24] Sheikholeslami, M., \& Shamlooei, M. (2017). Fe 3 O 4-H 2 O nanofluid natural convection in presence of thermal radiation. International Journal of Hydrogen Energy, 42(9), 5708-5718.

[25] Sheikholeslami, M. (2017). Magnetic field influence on nanofluid thermal radiation in a cavity with tilted elliptic inner cylinder. Journal of Molecular Liquids, 229, 137-147.

[26] Sheikholeslami, M., \& Shehzad, S. A. (2017). Thermal radiation of ferrofluid in existence of Lorentz forces considering variable viscosity. International Journal of Heat and Mass Transfer, 109, 82-92.

[27] Sheikholeslami, M., Hayat, T., \& Alsaedi, A. (2017). Numerical study for external magnetic source influence on water based nanofluid convective heat transfer. International Journal of Heat and Mass Transfer, 106, 745-755. [28] Sheikholeslami, M., \& Rokni, H. B. (2017). Numerical modeling of nanofluid natural convection in a semi annulus in existence of Lorentz force. Computer Methods in Applied Mechanics and Engineering, 317, 419-430.

[29] Malik, S., \& Nayak, A. K. (2017). MHD convection and entropy generation of nanofluid in a porous enclosure with sinusoidal heating. International Journal of Heat and Mass Transfer, 111, 329-345.

[30] Sheikholeslami, M. (2015). Effect of uniform suction on nanofluid flow and heat transfer over a cylinder. Journal of the Brazilian Society of Mechanical Sciences and Engineering, 37(6), 1623-1633.

[31] Sheikholeslami, M. (2016). CVFEM for magnetic nanofluid convective heat transfer in a porous curved enclosure. European Physical Journal Plus, 131(11).

[32] Sheikholeslami, M. (2017). Numerical simulation of magnetic nanofluid natural convection in porous media. Physics Letters A, 381(5), 494-503.

[33] Sheikholeslami, M., \& Ganji, D. D. (2017). Numerical approach for magnetic nanofluid flow in a porous cavity using CuO nanoparticles. Materials \& Design, 120, 382-393. 
[34] Sheikholeslami, M., \& Bhatti, M. M. (2017). Forced convection of nanofluid in presence of constant magnetic field considering shape effects of nanoparticles. International Journal of Heat and Mass Transfer, 111, 1039-1049. [35] Sheikholeslami, M. (2017). CuO-water nanofluid free convection in a porous cavity considering Darcy law. European Physical Journal Plus, 132(1).

[36] Sheikholeslami, M. (2017). Numerical simulation of magnetic nanofluid natural convection in porous media. Physics Letters A, 381(5), 494-503.

[37] Sheikholeslami, M. (2017). Influence of magnetic field on nanofluid free convection in an open porous cavity by means of Lattice Boltzmann method. Journal of Molecular Liquids, 234, 364-374.

[38] Sheikholeslami, M. (2017). Magnetohydrodynamic nanofluid forced convection in a porous lid driven cubic cavity using Lattice Boltzmann method. Journal of Molecular Liquids, 231, 555-565

[39] Sheikholeslami, M. (2017). Influence of Lorentz forces on nanofluid flow in a porous cylinder considering Darcy model. Journal of Molecular Liquids, 225, 903-912.

[40] Sheikholeslami, M., \& Bhatti, M. M. (2017). Forced convection of nanofluid in presence of constant magnetic field considering shape effects of nanoparticles. International Journal of Heat and Mass Transfer, 111, 1039-1049. [41] Sheikholeslami, M., \& Shehzad, S. A. (2017). Magnetohydrodynamic nanofluid convection in a porous enclosure considering heat flux boundary condition. International Journal of Heat and Mass Transfer, 106, 12611269.

[42] Sheikholeslami, M., \& Zeeshan, A. (2017). Analysis of flow and heat transfer in water based nanofluid due to magnetic field in a porous enclosure with constant heat flux using CVFEM. Computer Methods in Applied Mechanics and Engineering, 320, 68-81.

[43] Sheikholeslami, M. (2017). Lattice Boltzmann method simulation for MHD non-Darcy nanofluid free convection. Physica B: Condensed Matter, 516, 55-71.

[44] Oztop, H. F., Selimefendigil, F., Abu-Nada, E., \& Al-Salem, K. (2016). Recent developments of computational methods on natural convection in curvilinear shaped enclosures. Journal of Thermal Engineering, 2(2), 693-698.

[45] Lazarus, G., Roy, S., Kunhappan, D., Cephas, E., \& Wongwises, S. (2015). Heat transfer performance of silver/water nanofluid in a solar flat-plate collector. Journal of Thermal Engineering, 1(2), 104-112.

[46] Birkman, H. C. (1952). The viscosity of concentrated suspensions and solution. The Journal of Chemical Physics, 20, 571.

[47] Maxwell-Garnett, J.C, (1904). Colors in metal glasses and in metallic films. Philosophical Transactions of the Royal Society A, 203, 385-420.

[48] Fasshauer, G.E. Meshfree Approximation Methods with Matlab; World Scientific Publications, Singapore, 2007.

[49] Fasshauer, G.E.; McCourt, M. Kernel-based Approximation Methods using MATLAB; World Scientific Publications, Singapore, 2015.

[50] Ramakrishna, D., Basak, T., Roy, S., \& Pop, I. (2012). Numerical study of mixed convection within porous square cavities using Bejan's heatlines: Effects of thermal aspect ratio and thermal boundary conditions. International Journal of Heat and Mass Transfer, 55(21-22), 5436-5448.

[51] De Vahl Davis, G. (1983). Natural convection of air in a square cavity: A bench mark numerical solution. International Journal for Numerical Methods in Fluids, 3(3), 249-264. 